\title{
Time-resolved CARS studies of vibrational coherences in the condensed phase: $\mathbf{I}_{2}$ in solid krypton
}

\author{
Michael Karavitis ${ }^{1}$, Ilya Goldschleger ${ }^{1}$, V. Ara Apkarian ${ }^{1}$, and Takayuki Kumada ${ }^{2}$ \\ ${ }^{1}$ Department of Chemistry, University of California, Irvine, CA 92697-2025, USA \\ E-mail: aapkaria@uci.edu \\ ${ }^{2}$ Advanced Science Research Center, Japan Atomic Energy Research Institute, Tokai, \\ Ibaraki 319-1195, Japan \\ E-mail: kumada@popsvr.tokai.jaeri.go.jp
}

\begin{abstract}
Vibrational dephasing in the prototypical system of $\mathrm{I}_{2}$ isolated in solid $\mathrm{Kr}$ is studied using TRCARS. Decay $|0><v|$ of coherences for $v=1-19$, as a function of temperature $(T=6-45 \mathrm{~K})$, are extracted and analyzed.
\end{abstract}

\section{Introduction}

Time-resolved, electronically resonant, parametric four-wave mixing processes allow detailed preparation, manipulation, and interrogation of molecular rovibronic coherences. We implement this tool in the present to carry out systematic studies of vibrational dephasing of molecular iodine isolated in solid $\mathrm{Kr}$. Using ultrashort laser pulses with adjustable time-bandwidth profiles, we extract $v$ dependent rates of dephasing and dissipation for $v=1-19$, as a function of temperature for $T=6-45 \mathrm{~K}$.

\section{Experimental}

The experiments are carried out on thin films of $\mathrm{I}_{2} / \mathrm{Kr}$, using three $25-100 \mathrm{fs}$ laser pulses from two home-built non-collinear optical parametric amplifiers (NOPA) pumped by a $1 \mathrm{kHz}$ regeneratively amplified Ti:Sapphire laser. Intensities of time-resolved coherent anti-stokes Raman scattering (TR-CARS) from the sample are measured for coincident pump $(\mathrm{P})$ and Stokes $(\mathrm{S})$ beams, as a function of probe $\left(\mathrm{P}^{\prime}\right)$ time.

\section{Results and Discussion}

The measurements are restricted to the $P^{(0,3)}$ component of the third order polarization, in which the three input fields act on the state ket (bra) while the bra (ket) evolves field free [1]. With coincident pump (P) and Stokes (S) pulses, the 
prepared vibrational coherence corresponds to the Raman packet is created on the ket:

$$
\left|\varphi^{(2)}\left(k_{P}-k_{S} ; t\right) X \varphi^{(0)}\right|=e^{i\left(k_{P}-k_{S}\right) r} \sum_{v} c_{v} e^{-i E_{v} t / \hbar}|v X 0| e^{i E_{0} t / \hbar}
$$

The time-delayed probe pulse (P') interrogates the vibrational coherence, with the anti-Stokes radiation along $\mathrm{k}_{\mathrm{AS}}=\mathrm{k}_{\mathrm{P}}-\mathrm{k}_{\mathrm{S}}+\mathrm{k}_{\mathrm{P}}$, serving as signal. The coherence will decay by coupling to the lattice phonons, by pure dephasing $T_{2}{ }^{*}$ and dissipation $T_{1}$ processes. The dephasing of a vibrational wave packet composed of an $N$-state superposition will be described by $N$-dephasing rate constants $\gamma_{v}$, describing the loss of amplitude correlation between state $v$ and the ground vibrational level $(v=0)$. After tracing over the bath, the decay of the reduced system density can be described as:

$$
\rho_{v, v^{\prime}}=\sum_{v, v^{\prime}} 2 c_{v} c_{v^{\prime}} \cos \left(\omega_{v, v^{\prime}} t+\phi_{v, v^{\prime}}\right) \exp \left[-\left(\gamma_{v}+\gamma_{v^{\prime}}\right) t\right]
$$

TR-CARS signals obtained with a Stokes shift centered on $v=17$ and 18 are shown in Fig. 1. The extracted $T$-dependent values of $\gamma(v=17,18)$ are also plotted. $\gamma$ is independent of $T$ below $10 \mathrm{~K}$, but it steeply increases with $T$ above. Fig. 2 shows $\gamma$ as a function of $v$. The $v$-dependence is essentially linear at $7 \mathrm{~K}$, whereas it can be fit with a quadratic dependence on $v$ at $34 \mathrm{~K}$. The entire data set can be well represented by the functional form:

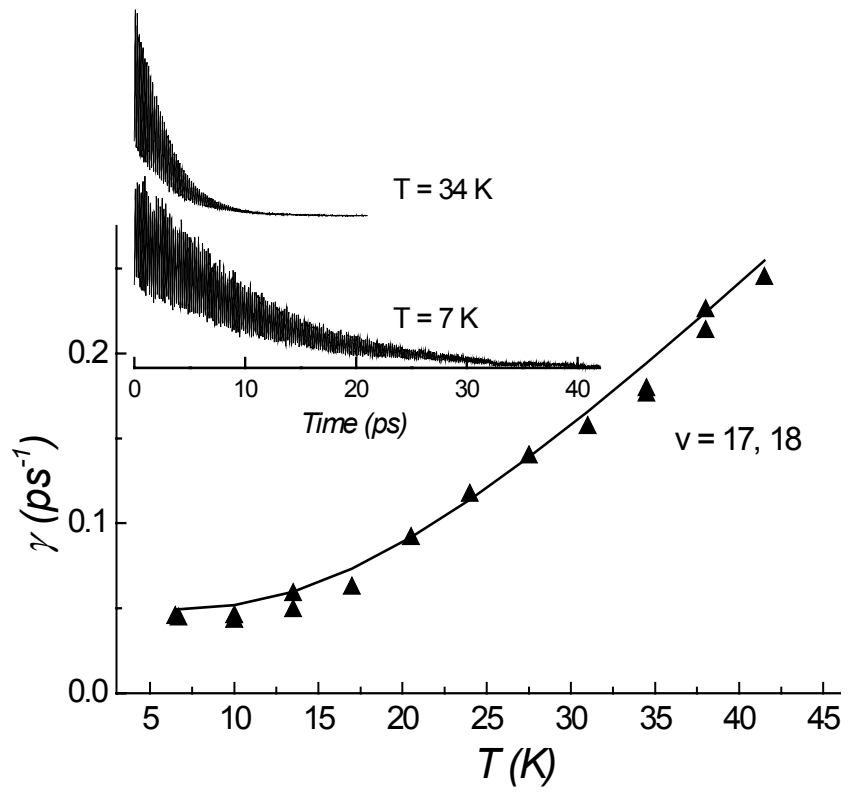

Fig. 1. Temperature dependence of vibrational dephasing rate for a wavepacket centered at $v=17,18$. The data points are extracted from the decay of D.C. component of TRCARS signal. The solid line represents the best fit to experimental data using Eq. (3). The inset shows two representative TRCARS signals obtained at 7 and $34 \mathrm{~K}$. The dephasing rates of the two signals vary by more than factor of three. 


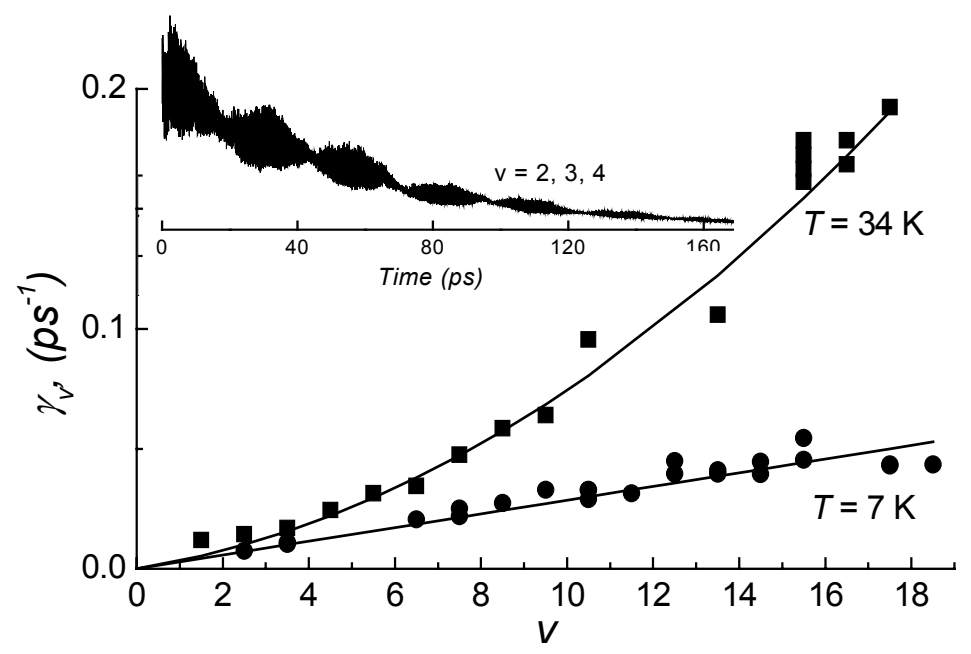

Fig. 2. Dephasing rate as a function of vibrational quantum number measured at 7 and 34 $\mathrm{K}$. At $7 \mathrm{~K}$, dephasing rate displays a nearly linear dependence on $v$, while at $34 \mathrm{~K}$, this dependence nominally quadratic. The inset illustrates a time domain signal containing a superposition of vibrational states $v=2-4$. This signal exhibits nodes separated by $26 \mathrm{ps}$, corresponding to the anharmonic shift between adjacent vibrational levels.

$$
\gamma(v, T)=\frac{v}{\tau_{1}}+\frac{v^{2}}{\tau_{2}} \frac{1}{e^{\theta / T}-1}
$$

where $\tau_{1}=355 \pm 10 \mathrm{ps}, \tau_{2}=560 \pm 70 \mathrm{ps}$, and $\theta=54 \pm 4 \mathrm{~K}$, to be compared with the Debye temperature of $72 \mathrm{~K}$ of solid $\mathrm{Kr}$. For $T<10 \mathrm{~K}$, when the high frequency phonons are not populated, the decay becomes $T$-independent, suggesting dissipation by the spontaneous creation of phonons. Note that to bridge the vibrational energy gap of the molecule, the dissipation must involve the simultaneous creation of four phonons. On the other hand, for $T>10 \mathrm{~K}$, pure dephasing is activated by the thermally populated phonons. A density of states analysis for phonon projections on the molecular coordinate is used to identify the nature of the pseudo local phonons responsible for the observed dephasing. Given the relatively well known interaction potentials between guest and host, quantitative treatments of this system can be expected.

\section{References}

1 M. Karavitis, R. Zadoyan, V. A. Apkarian, in Journal of Chemical Physics, Vol.114, 4131, 2001.

2 M. Karavitis, V. A. Apkarian, "Vibrational Coherence of $\mathrm{I}_{2}$ in Solid Kr, in Journal of Chemical Physics, Vol.120, 292, 2004. 